\title{
Corporate Governance, Regulierung und Rechnungslegung
}

\author{
Ralf Ewert • Hans-Ulrich Küpper
}

\section{Editorial}

Dieses Sonderheft enthält Vorträge und Koreferate der 36. Sitzung des Ausschusses „Unternehmensrechnung" im Verein für Socialpolitik, die vom 29.-30. April 2011 an der Universität Hannover zum Thema „Corporate Governance, Regulierung und Rechnungslegung" stattfand.

Corporate Governance und Regulierung sind einerseits zentrale Determinanten für die Funktionen und die Ausgestaltung der Rechnungslegung, andererseits bilden die Zahlen der Rechnungslegung eine wichtige Basis, um Vorstellungen im Bereich der Corporate Governance und der Regulierung zu erfüllen. Die Beiträge und Koreferate in diesem Heft beleuchten diese Thematik aus unterschiedlichen Blickrichtungen.

Hanno Merkt behandelt die Rolle des Bilanzrechts in der aktuellen Corporate Governance Diskussion und zeigt, dass aus den neueren Entwicklungen im Bereich der Corporate Governance ein veränderter Fokus auf die Rechnungslegung resultiert, der weniger die Informations- und Transparenzfunktion als die Funktion der Selbstinformation bzw. Selbstrechenschaft in den Mittelpunkt rückt. Dies wird demonstriert am Beispiel der Berichterstattung über das Risikomanagementsystem und der Prüfung des Jahresabschlusses mit dem Wechselspiel zwischen Prüfungsausschuss und Gesamtaufsichtsrat.

Die Prüfung des Jahresabschlusses wird im Beitrag von Christopher Bleibtreu und Ulrike Stefani unter dem Aspekt des von der EU-Kommission im Oktober 2010 veröffentlichten Grünbuchs zur Abschlussprüfung diskutiert. Dort findet sich unter anderem der Vorschlag, Prüfung und Beratung beim gleichen Mandanten sehr weitgehend voneinander zu trennen. Die Autoren untersuchen auf Basis einer modellanalytischen Vorgehensweise die Interdependenz zwischen dem Verbot von Prüfung und Beratung, den Anreizen zum

(C) Gabler-Verlag 2012

Prof. Dr. R. Ewert ( $\square)$

Institut für Unternehmensrechnung und Wirtschaftsprüfung,

Karl-Franzens-Universität Graz, Universitätsstr. 15/F1, 8010 Graz, Österreich

E-Mail: ralf.ewert@uni-graz.at

Prof. Dr. Dr.h.c. H.-U. Küpper

Institut für Produktionswirtschaft und Controlling, LMU München,

Ludwigstraße 28, 80539 München, Deutschland

E-Mail: kuepper@bwl.lmu.de 
Markteintritt von Prüfungsgesellschaften und der Struktur des Prüfungsmarktes. Die Ergebnisse sind überraschend, es ist zum Beispiel völlig offen, ob eine Trennung von Prüfung und Beratung zur Verbesserung der Unabhängigkeit führt.

Christoph Kaserer, Marc Steffen Rapp und Oliver Trinchera gehen auf Basis einer empirischen Untersuchung der Frage nach, welche Ausschüttungskonsequenzen für das Steuersenkungsgesetz 2001 identifiziert werden können. Mit dieser Gesetzgebung wurde in Deutschland das bis dahin geltende Anrechnungsverfahren bei der Körperschaftsteuer durch das sogenannte Halbeinkünfteverfahren ersetzt. Die Autoren finden, dass sich nach Einführung dieses Gesetzes sowohl eine verminderte Bereitschaft zur Dividendenzahlung als auch eine reduzierte Höhe der Ausschüttungen nachweisen lässt. Allerdings hat es bei Unternehmen, in denen das Management bedeutende Stimmrechte hat, keine Änderung des Ausschüttungsverhaltens gegeben, und zwar unabhängig davon, ob es andere einflussreiche Anteilseigner (wie etwa institutionelle Investoren) gibt. Die Autoren folgern, dass die Ausschüttungspolitik vornehmlich durch die Interessen der Insider bestimmt wird.

Robert Göx und Alexis Kunz widmen sich in ihrem Beitrag der aktuellen Problematik des „Say on Pay“, also der Möglichkeit von Aktionären, über die Höhe und Struktur der Vergütung des Top-Managements abzustimmen. Derartige Rechte können sehr unterschiedlich ausgestaltet sein, je nachdem, ob das Votum der Aktionäre ex ante oder ex post erfolgt, und ob es eher konsultativen Charakter hat oder eine echte Bindungswirkung entfaltet. Die Autoren zeigen auf Basis eines grundlegenden Prinzipal-Agenten-Modells, dass die ökonomischen Konsequenzen dieser unterschiedlichen Varianten deutlich differieren und runden ihre Darstellung mit einem Überblick über empirische und experimentelle Belege $a b$.

Der Beitrag von Dirk Hachmeister, Niklas Lampenius und Martin Stähle behandelt zwei Systeme der Rechnungslegung, das „Ideal Cost Accounting“ (ICA) einerseits als ein System, in dem Kosten in perfekter Beziehung zu den Cashflow-Beiträgen der jeweiligen Perioden stehen und Produkte mit ihren Grenzkosten bewertet werden, und das „Ideal Value Accounting“ (IVA) andererseits als ein System, in dem die Buchwerte in der Rechnungslegung grundsätzlich den Marktwerten bzw. Barwerten entsprechen. Sie untersuchen, wie sich diese Systeme hinsichtlich ihrer Konsequenzen für Entscheidungsnützlichkeit und Stewardship verhalten. Die modellanalytische Untersuchung basiert auf einem Szenario, in dem ein Unternehmen im Zeitablauf überlappende und wertschaffende Projekte realisiert. Die Autoren zeigen, dass ICA grundsätzlich gut abschneidet, während für IVA - je nach Kontext - Probleme identifiziert werden können.

Franz Wagner und Reinhard Sill analysieren die Einbeziehung von Ertragsteuern in Kalküle der Kostenrechnung. Dabei geht es einerseits um Anwendungen regulatorischer Art (etwa den Ansatz der Gewerbesteuer im Rahmen von LSP-Preiskalkulationen oder Strom- und Gasnetzentgeltverordnungen), andererseits um den Ansatz in kostenrechnerischen Entscheidungskalkülen. Es zeigt sich, dass Ertragsteuern in der „Lehrbuchliteratur“ teils signifikant anders behandelt werden als in der Praxis. Während die Vorgehensweise von Unternehmen eher mit der ökonomischen Bedeutung für kostenrechnerische Entscheidungskalküle vereinbar ist, scheinen Kammern, Verbände und die Lehrbuchliteratur vornehmlich nach tradierten steuerrechtlichen Aspekten zu urteilen, trotz der Tatsache, dass sich die diesbezügliche Einschätzung nach der Unternehmenssteuerreform 2008 eigentlich verändert haben müsste. 
Erneut zeigt sich, dass die Arbeit des Ausschusses „Unternehmensrechnung“ im Verein für Socialpolitik sowohl aus inhaltlicher als auch aus methodischer Sicht ein breites Spektrum abdeckt. Die einzelnen Beiträge werden jeweils durch Kommentare ergänzt, die nicht nur eine intuitive Erklärung der Resultate und eine Einordnung in die relevante Literatur, sondern vielfach auch weiterführende Überlegungen der Koreferenten enthalten, denen wir für die damit verbundene Mühe herzlich danken. Wir sind davon überzeugt, dass dieses Heft eine spannende Lektüre mit Beiträgen bietet, die international kompetitive Forschung beinhalten. 